\title{
Die Konzentrations-Abhängigkeit des Sedimentations-Koeffizienten von Transferrinen
}

\author{
Von Andreas Mahling \\ Aus der Behringwerke AG., Marburg/Lahn (wissensch. Leiter: Prof. Dr. H. E. Schultze) \\ Veröffentlicht anläßlich des 100jährigen Jubiläums der Farbwerke Hoechst AG. am 11. Januar 1963 \\ (7. Naturforschg. 18 b, 1-3 [1963]; eingegangen am 13. Juli 1962)

\begin{abstract}
Zwei verschiedene Human-Transferrine werden in Einzelläufen mit verschiedenen Konzentrationen und in einer Überschichtungszelle mit einer Differentialgrenzschicht in der Ultrazentrifuge untersucht. Die Werte, die man in der Überschichtungszelle für die auf 0 extrapolierte Sedimentationskonstante $s_{0}$ und für die Steigungskonstante $k$ bei linearer Konzentrations-Abhängigkeit erhält, entsprechen innerhalb der Vertrauensgrenzen den aus den Einzelläufen berechneten Werten.
\end{abstract}

Die von Hersh und Schachmann ${ }^{1}$ beschriebene Überschichtungszelle, mit der man während eines Ultrazentrifugenlaufes eine Lösung mit einer anderen überschichten kann, wobei zwischen ihnen eine scharfe Grenzschicht entsteht, gestattet die Erzeugung einer Konzentrations- oder Differentialgrenzschicht, indem man eine konzentriertere $\left(c_{2}\right)$ mit einer verdünnteren $\left(c_{1}\right)$ Lösung überschichtet. Die Aufnahmen des Brechungsindexgradienten längs der Zellkoordinate weisen dann zwei Peaks auf (Abb. 1), aus deren Wanderungsgeschwindigkeiten auf die Konzentrations-Abhängigkeit des SedimentationsKoeffizienten geschlossen werden kann.

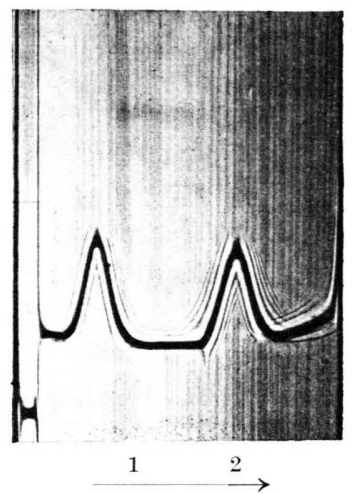

Abb. 1. Differentialgrenzschicht. Transferin 1870: Aufnahme nach $30 \mathrm{~min}$ Laufzeit. Konzentrationsänderung am 1. Peak: $0 \rightarrow 0,456 \mathrm{~g} / \mathrm{dl}$, am 2. Peak : $0,456 \mathrm{~g} / \mathrm{dl} \rightarrow 0,916 \mathrm{~g} / \mathrm{dl}$.

Es gilt nämlich ${ }^{1}$ für den Sedimentations-Koeffizienten $s_{\mathrm{D}}$ der Differentialgrenzschicht:

$$
s_{\mathrm{D}}=\frac{c_{1} s_{1}-c_{2} s_{2}}{c_{1}-c_{2}} .
$$

1 R. T. Hersh u. M. K. Schachmann, J. Amer. chem. Soc. 77, 5228 [1955].

2 C. G. Holmberg u. C. B. Laurell, Acta chem. scand. 1, 944 [1947].
Wenn man eine lineare Beziehung zwischen Konzentration $c$ und Sedimentations-Koeffizienten $s$ nach

$$
s=s_{0}(1-k c) \quad s_{0}, k=\text { const. }
$$

voraussetzen darf, so folgt aus (1) und (2)

$$
s_{\mathrm{D}}=s_{0}\left[1-k\left(c_{1}+c_{2}\right)\right],
$$

d.h., die Differentialgrenzschicht bewegt sich mit einer Geschwindigkeit, die der Summe der Konzentrationen in beiden Lösungen entspricht. Der sich vom Meniskus nach der Überschichtung ablösende Peak wandert mit einem Sedimentations-Koeffizienten $s_{1}$, entsprechend der Konzentration $c_{1}$, so daß man über zwei Wertepaare $s_{1}, c_{1}$ und $s_{\mathrm{D}}, c_{1}+c_{2}$ verfügt, aus denen sich die Parameter $s_{0}$ und $k$ der Gl. (2) mit Hilfe der Formeln

$$
\begin{aligned}
& s_{0}=\left(s_{1}-s_{\mathrm{D}}\right) \cdot \varphi+s_{1}, \\
& k=\frac{s_{1}-s_{\mathrm{D}}}{s_{0}} \frac{1}{c_{2}}
\end{aligned}
$$

mit $\varphi=c_{1} / c_{2}<1$ berechnen lassen.

Zur Prüfung der Gln. (4) und (5) wurde das von Holmberg und Laurell ${ }^{2}$ und, von ihnen unabhängig, auch von $\mathrm{S}_{\mathrm{CHADE}}{ }^{3}$ im Humanplasma entdeckte, eisenhaltige Transferrin (Siderophilin) herangezogen.

Von zwei Chargen (Laborpräparate) ${ }^{4}$ mit den Nummern 1870 und 1227/34 wurden verschieden konzentrierte Lösungen in einem Phosphatpuffer vom $p_{\mathrm{H}}\left(20,0{ }^{\circ} \mathrm{C}\right)=6,78$ und einem spezifischen Widerstand $r\left(20,0{ }^{\circ} \mathrm{C}\right)=44,5 \mathrm{Ohm} \cdot \mathrm{cm}$, der 0,02-m. $\mathrm{NaH}_{2} \mathrm{PO}_{4} ; 0,03-m . \mathrm{Na}_{2} \mathrm{HPO}_{4}$ und $0,20-m . \mathrm{NaCl}$ enthielt, konventionellen Ultrazentrifugenläufen unterworfen. $p \mathrm{H}$-Werte und Widerstände der Lösun-

3 A. L. Schade, R. W. Reinhardt u. H. Levy, Acta biochim. polon. 20, 170 [1949].

4 H. E. Schultze, I. Göllner, K. Heide, M. Schönenberger u. G. Sсншіск, Z. Naturforschg. 10 b, 463 [1955]. 
gen unterschieden sich nur im Rahmen des Zufalles von den entsprechenden Werten des reinen Puffers. Die Konzentrationen waren teils durch Einwaage unter Berücksichtigung des Wassergehaltes, teils durch Ausplanimetrieren der Gradientenkurven bekannt.

Die Nummer 1870 enthielt $990 \gamma \mathrm{Fe} / \mathrm{g}$ und war neuraminsäurefrei; der Nummer 1227/34 war das Eisen mit Hilfe von Äthylendiamintetraessigsäure entzogen worden, während der Neuraminsäuregehalt dem nativen Zustande entsprach.

Die Umdrehungsgeschwindigkeit der Ultrazentrifuge (Spinco, Modell E) betrug in allen Fällen $59780 \mathrm{U} / \mathrm{min}$, die Temperatur $20,0 \pm 0,5^{\circ} \mathrm{C}$.

Die photographischen Aufnahmen der Brechungsindexgradienten-Kurven wurden unter einem Meßmikroskop mit einer Genauigkeit von $\pm 0,001 \mathrm{~cm}$ vermessen. Die Sedimentations-Koeffizienten ermittelte man für jeden Lauf mittels eines Rechenautomaten nach der Methode der kleinsten Quadrate unter Voraussetzung einer linearen Beziehung zwischen dem Logarithmus des Abstandes Peak - Rotationszentrum und der Laufzeit.

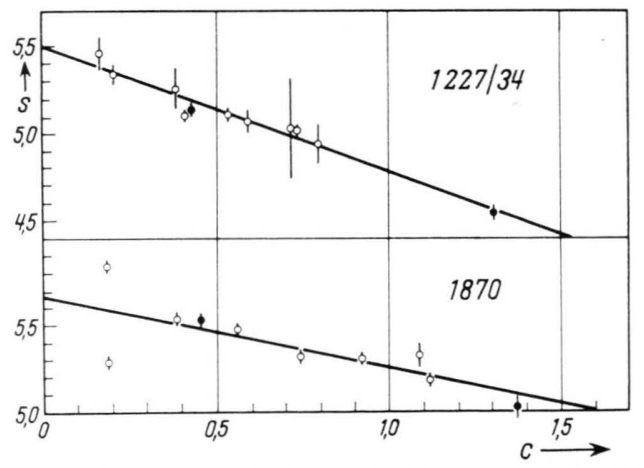

Abb. 2. Die Abhängigkeit des Sedimentations-Koeffizienten von der Konzentration. $[s]=$ Sved, $[c]=\mathrm{g} / \mathrm{dl}$.

Abb. 2 zeigt die auf Wasser umgerechneten Sedimentations-Koeffizienten mit ihren 95\%-Vertrauensbereichen in $\mathrm{S} v$ edber g-Einheiten auf den Ordinaten und den Konzentrationen in $\mathrm{g} / \mathrm{dl}$ auf den $\mathrm{Ab}$ szissen. Die aus den Läufen mit der Differentialgrenzschicht erhaltenen Wertepaare sind durch ausgefüllte Kreise gekennzeichnet. Die ausgezogenen Geraden wurden nach der Methode der kleinsten Quadrate berechnet und entsprechen den Gleichun. gen:

$$
\begin{gathered}
s=(5,628 \pm 0,108)[1-(0,074 \pm 0,049) \cdot c], \\
\text { Nr. } 1870 \\
s=(5,505 \pm 0,036)[1-(0,131 \pm 0,024) \cdot c] . \\
\text { Nr. } 1227 / 34
\end{gathered}
$$

Die angegebenen Fehlergrenzen sind wiederum die 95\%-Vertrauensbereiche.

Die Läufe in der Überschichtungszelle ergaben:

$$
\begin{gathered}
s=(5,797 \pm 0,071)[1-(0,097 \pm 0,016) \cdot c], \\
\text { Nr. } 1870
\end{gathered}
$$$$
s=(5,428 \pm 0,067)[1-(0,124 \pm 0,012) \cdot c] .
$$

In diesen Fällen wurden die 95\%-Vertrauensbereiche mittels des Fehlerfortpflanzungsgesetzes unter Vernachlässigung der Varianzen für $\varphi$ und $c_{2}$ aus den Gl. (4) und (5) berechnet.

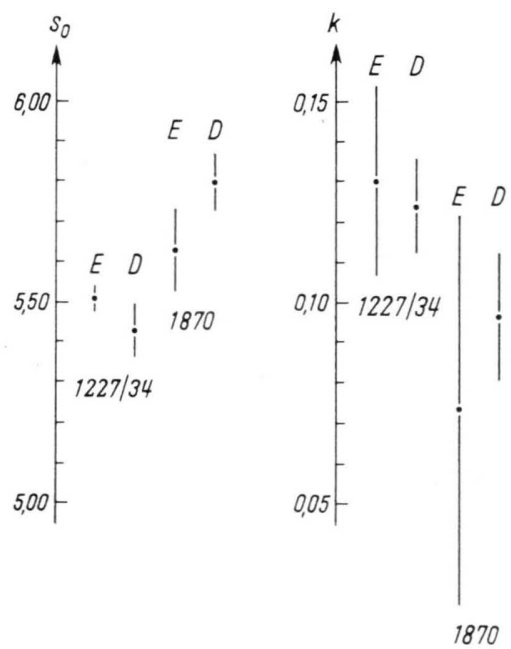

Abb. 3. $s_{0}$ - und $k$-Werte mit ihren 95\%-Vertrauensbereichen $\left[s_{0}\right]=$ Sved. $[k]=\mathrm{dl} / \mathrm{g}$.

Auf der Abb. 3 sind $s_{0}$ - und $k$-Werte mit ihren Vertrauensgrenzen graphisch dargestellt, worin die Chargen durch ihre Nummern und die Läufe durch die Buchstaben E (Einzelläufe) und D (Differentialgrenzschicht) gekennzeichnet sind. Die Streuungen sind bei den Einzelläufen der Nr. 1870 am größten und unterscheiden sich auch im F is he r' schen F-Test signifikant von den Streuungen der Nr. 1227/34. Da andere Faktoren, die die Sedimentation beeinflussen, wie Temperatur, Viskosität, Dichte usw. bei beiden Versuchsreihen den gleichen Varianzen unterworfen waren, muß man annehmen, daß die vergrößerte Streuung der Nr. 1870 eine Eigenschaft der gelösten Substanz selbst ist, indem, vielleicht auf Grund der fehlenden Neuraminsäure, ihre Stabilität verringert und der Reibungsfaktor stärker zufälligen Veränderungen ausgesetzt ist.

Es bedarf eines sehr viel größeren Aufwandes. um solche zufälligen Veränderungen bei mehreren Einzelläufen auszuschalten, als bei einem einzigen 
Lauf mit einer Differentialgrenzschicht, weshalb diese Werte in sich zuverlässiger sind, zumal apparative Faktoren, wie Schwankungen der Drehzahl und der Temperatur zwangsläufig dieselben sind. Das bedeutet jedoch keine Genauigkeitssteigerung für die Absolutwerte, weil hierbei auch noch Fragen des Ausgangsmaterials und seiner Präparationstechnik eine dominierende Rolle spielen können.

\title{
Aluminum hydride derivatives containing Al-S bond
}

\author{
By Walter Marconi, Alessandro Mazzei, Flavio Bonati and Marcello de Maldè \\ From the Laboratori Riuniti Studi e Ricerche, S. Donato (Milan) \\ (Z. Naturforschg. 18 b, 3-5 [1963]; eingegangen am 30. August 1962)
}

\begin{abstract}
The synthesis of aluminum hydrides derivatives containing Al-S bond, obtained reacting a thiol with $\mathrm{AlH}_{3}$ and its derivatives, are reported.

According to the poor electron donor properties of sulphur in thiols, such alanes are complexed with trimethyl-amine and sometimes they are associated through hydrogen bridges in benzene solution.
\end{abstract}

By treating some active hydrogen compounds, in order to study the substitution reaction of one or more hydrogen atoms with aluminum hydrides complexed with trialkyl-amines, compounds with $\mathrm{Al}-\mathrm{N}^{1-3}, \mathrm{Al}-\mathrm{Cl}^{3}, \mathrm{Al}-\mathrm{P}^{4}$ bond were obtained.

It is further known that $\mathrm{Al}-\mathrm{S}$ bond is obtained by reaction of aluminum alkyls with mercaptans ${ }^{5}$ as follows:

$$
\left(\mathrm{AlMe}_{3}\right)_{2}+2 \mathrm{HSMe}=\left(\mathrm{AlMe}_{2} \mathrm{SMe}\right)_{2}+2 \mathrm{CH}_{4}
$$

After considering the reaction of aluminum hydrides with secondary amines, we examined a similar reaction with aliphatic and aromatic mercaptans. Mercapto alanes $\mathrm{AlH}_{2} \mathrm{SR}: \mathrm{NMe}_{3}$, $\mathrm{AlH}(\mathrm{SR})_{2}: \mathrm{NMe}_{3}, \mathrm{Al}(\mathrm{SR})_{3}: \mathrm{NMe}_{3}$ (where $\mathrm{R}=$ $-n \mathrm{C}_{3} \mathrm{H}_{7}$ or $-\mathrm{C}_{6} \mathrm{H}_{5}$ ) or substituted mercaptoalanes such as

$$
\operatorname{AlHCl}(\mathrm{SR}): \mathrm{NMe}_{3} \text { and } \mathrm{AlH}\left(\mathrm{NC}_{5} \mathrm{H}_{10}\right)(\mathrm{SR})
$$

were thus obtained, according to the following quantitative reactions:

$$
\mathrm{AlH}_{3}: \mathrm{NMe}_{3}+n \mathrm{HSR}=\mathrm{AlH}_{3-\mathrm{n}}(\mathrm{SR})_{\mathrm{n}}: \mathrm{NMe}_{3}+n \mathrm{H}_{2}
$$

where:

$$
\begin{gathered}
n=1,2,3 ; \quad \mathrm{Me}=-\mathrm{CH}_{3} \\
\mathrm{AlH}_{2} \mathrm{Cl}: \mathrm{NMe}_{3}+\mathrm{HSR}=\mathrm{AlHCl}(\mathrm{SR}): \mathrm{NMe}_{3}+\mathrm{H}_{2} \\
\mathrm{AlH}_{3}: \mathrm{NMe}_{3}+\mathrm{HNC}_{5} \mathrm{H}_{10}+\mathrm{HSR} \\
=\mathrm{AlH}\left(\mathrm{NC}_{5} \mathrm{H}_{10}\right)(\mathrm{SR})+\mathrm{NMe}_{3}+2 \mathrm{H}_{2}
\end{gathered}
$$

1 E. Wiberg and A. May, Z. Naturforschg. 10 b, 234 [1955].

2 J. K. Ruff and M. F. Hawthorne, J. Amer. chem. Soc. 82, 2141 [1960]; 83, 535 [1961].

3 W. Marconi, A. Mazzei, F. Bonati and M. de Maldè, Gazz. chim. ital., in press. or indirectly:

$$
2 \mathrm{AlH}_{3}: \mathrm{NMe}_{3}+\mathrm{Al}(\mathrm{SR})_{3}: \mathrm{NMe}_{3}=3 \mathrm{AlH}_{2} \mathrm{SR}: \mathrm{NMe}_{3}
$$

In this case confirmation that the reaction took place is obtained by considering the wave length shift of the $\mathrm{Al}-\mathrm{H}$ band in the $\mathrm{I}$. R. spectrum of starting material and reaction products.

The mercapto-alanes are colorless oily liquids or waxy solids with low melting point, and have to be handled under nitrogen because of their tendency to react with moisture and oxygen. They cannot be purified by vacuum distillation or sublimation; in fact under such conditions they undergo thermal decomposition.

Compounds containing thio- $n$-propyl group are soluble in diethyl-ether and benzene, while those containing tiophenyl group are difficultly soluble.

Cryoscopic molecular weight determinations in benzene show a degree of association of 1.55 for $\mathrm{AlH}_{2} \mathrm{~S}\left(n \mathrm{C}_{3} \mathrm{H}_{7}\right): \mathrm{NMe}_{3} \quad(25.5$ and $18.5 \mathrm{~g} / \mathrm{lt})$ and of 1.9 for $\mathrm{AlH}_{2}\left(\mathrm{SC}_{6} \mathrm{H}_{5}\right): \mathrm{NMe}_{3}(16 \mathrm{~g} / \mathrm{lt})$.

It is known that $\left(\mathrm{Me}_{2} \mathrm{AlSMe}\right)_{2}$ splits into two monomeric units on reaction with trimethyl-amine ${ }^{6}$. The fact that such compounds, although containing one mole of $\mathrm{NMe}_{3}$ per mole of aluminum (which was not possible to remove without decomposition) have a degree of association higher than 1, point

${ }^{4}$ A. B. Burg u. Mödritzer, J. inorg. nuclear. Chem. 13, 318 [1960].

5 J. M. Davidson and H. Brown, J. Amer. chem. Soc. 64, 316 [1942].

${ }^{6}$ G. E. Coates, "Organo-metallic Compounds", 2nd ed., page 140, J. Wiley, New York 1960. 